Draft Version July 10, 2020

Typeset using LATEX default style in AASTeX63

\title{
Global Energetics of Solar Flares. XI. Flare Magnitude Predictions of the GOES-Class
}

\author{
MARKus J. AschWANDEN ${ }^{1}$ \\ ${ }^{1}$ Solar and Stellar Astrophysics Laboratory (LMSAL), Palo Alto, CA 94304, USA
}

\begin{abstract}
In this study we determine scaling relationships of observed solar flares that can be used to predict upper limits of the GOES-class magnitude of solar flares. The flare prediction scheme is based on the scaling of the slowly-varying potential energy $E_{p}(t)$, which is extrapolated in time over an interval of $\Delta t \leq 24 \mathrm{hrs}$. The observed scaling of the dissipated energy $E_{\text {diss }}$ scales with the potential field energy as $E_{\text {diss }} \propto E_{p}^{1.32}$. In addition, the observed scaling relationship of the flare volume, $V \propto E_{\text {diss }}^{1.17}$, the multi-thermal energy, $E_{t h} \propto V^{0.76}$, the flare emission measure $E M \propto E_{t h}^{0.79}$, the EM-weighted temperature $T_{w}$, and the GOES flux, $F_{8}(t) \propto E_{p}(t)^{0.92}$, allows us then to predict an upper limit of the GOES-class flare magnitude in the extrapolated time window. We find a good correlation (CCC $\approx 0.7)$ between the observed and predicted GOES-class flare magnitudes (in $172 \mathrm{X}$ and M-class events). This is the first algorithm that employs observed scaling laws of physical flare parameters to predict GOES flux upper limits, an important capability that complements previous flare prediction methods based on machine-learning algorithms used in space weather forecasting.
\end{abstract}

Keywords: Solar flares — soft X-rays

\section{INTRODUCTION}

Solar flare predictions, in particular for eruptive events that impact the heliospheric space weather, became an important area of research that may mitigate the health risk of astronauts in space, minimize power plant outages, and safeguard electronic equipment in the near-Earth space environment. An early warning system that could make short-term predictions within a time scale of 24 hours would be desirable. For the development of such a prediction tool we have to ask the question: What are the most important physical parameters that facilitate the prediction of energies dissipated in solar flares and space weather events? Observables used for previous flare prediction methods include: the modified Zurich sunspot classification, the penumbra type of the largest sunspot, types of sunspot distributions (Gallagher et al. 2002), high-gradient polarity-separation lines in line-of-sight magnetograms, the unsigned magnetic flux near the polarity-separation lines (Schrijver 2007), the effective connected magnetic field between unipolar magnetic areas in a flux-partitioned magnetogram (Georgoulis and Rust 2007), the length of the (neutral) polarity inversion line, the overall twist and shear of the non-potential magnetic field, the length segment over which the transverse magnetic field is strong (Falconer et al. 2003), the free magnetic energy (Falconer et al. 2011), the gradient-weighted inversion-line length (Mason and Hoeksema 2010; Cui et al. 2006), the unsigned current helicity, the total magnitude of the Lorenz force, the total photospheric magnetic free energy density, the total unsigned vertical current (Bobra and Couvidat 2015), the history of prior flaring (Falconer et al. 2012), the combination of UV brightenings, soft X-ray data, and vector magnetograph data (Nishizuka et al. 2017), synthesized photospheric and coronal data (Jonas et al. 2018), magnetic helicity injection in active regions (Tiwari et al. 2010), and measures of fractality, multi-fractality, and turbulence (Georgoulis 2012). This large arsenal of observables, which is still not capable to provide a fully satisfactory flare prediction method so far, indicates either that we have not yet identified the most relevant physical parameters, or that the measurement accuracy of the relevant physical parameters is insufficient. In both cases, machine-learning methods may not solve the problem (if the training data set does not contain the most relevant physical parameters), and thus we have to resort to more refined physical models. A benchmark test has been conducted during an inter-

Corresponding author: Markus J. Aschwanden

aschwanden@lmsal.com 
agency workshop on "all-clear" forecast, where the performance of a number of existing flare prediction algorithms was compared with common data sets from the Michelson Doppler Imager (MDI) (Scherrer et al. 1995) onboard the Solar and Heliospheric Observatory (SOHO) (Fleck et al. 1995), but it was found that no single method outperformed all others, similarly to the situation in climatological forecasting (Barnes et al. 2016).

In this study we develop a flare prediction method that is based on scaling relationships between magnetic field parameters (potential energy, non-potential energy, free energy, dissipated energy), geometric parameters (flare length scale, flare area, flare volume), temperature parameters (emission measure-weighted temperature), and energetic parameters (multi-thermal energy, emission measure, GOES flux). Most parameters have been previously measured in a series of papers on the global energetics of solar flares and coronal mass ejections (CME), in particular in Paper I (Aschwanden et al. 2014), Paper II (Aschwanden et al. 2015a), and Paper IX (Aschwanden 2019a). A fundamentally new aspect of this method developed here is the strategy to model the force-free coronal magnetic field with automatically traced coronal loop coordinates (e.g., Aschwanden 2016), which bypasses the problem of the non-forcefree photospheric magnetic field that is used in traditional magnetic field extrapolation methods (e.g., Wiegelmann et al. 2006). All the used observables and scaling relationships are based on data from the Helioseismic and Magnetic Imager (HMI) (Scherrer et al. 2012) and the Atmospheric Imaging Assembly (AIA) (Lemen et al. 2012) onboard the Solar Dynamics Observatory (SDO) (Pesnell et al. 2012), and from the Geostationary Orbiting Earth Satellites (GOES) (e.g., Lemen et al. 2004).

The contents of this study include a summary of the most relevant observational results (Section 2.1-2.4), modeling results of flare predictions in terms of GOES-class magnitudes (Section 2.5), a discussion and comparisons with previous flare prediction methods (Section 3), and conclusions (Section 4).

\section{DATA ANALYSIS AND RESULTS}

\subsection{Previous Observations and Parameter Measurements}

We use the same data set of solar flares presented in Paper I (Aschwanden et al. 2014) and Paper II (Aschwanden et al. 2015a), which includes all M- and X-class flares observed with the SDO (Pesnell et al. 2012) during the first 3.5 years of the mission (2010 June 1 to 2014 January 31). This selection provides 172 flare events with measurements of magnetic parameters (within a heliographic longitude range of $\left[-45^{\circ},+45^{\circ}\right]$, for which magnetic field modeling can be faciliated without too severe foreshortening effects near the solar limb), and 391 flare events for measurements of thermal parameters (for events at all longitudes $\left[-90^{\circ},+90^{\circ}\right]$ ).

We use the 45-s line-of-sight magnetograms from HMI/SDO and make use of all coronal EUV channels of AIA (Lemen et al. 2012) onboard the Solar Dynamics Observatory (SDO) (in the six wavelengths 94, 131, 171, 193, 211, $335 \AA$ ), which are sensitive to strong iron lines in the temperature range of $T \approx 0.6-16 \mathrm{MK}$. The spatial resolution is $\approx 1.6 "$ " (0.6" pixels) for AIA, and the pixel size of HMI is $0.5 "$.

The coronal magnetic field is modeled by using the line-of-sight magnetogram $B_{z}(x, y)$ from the Helioseismic and Magnetic Imager (HMI) (Scherrer et al. 2012) and (automatically detected) projected loop coordinates $[x(s), y(s)]$ in each EUV wavelength of AIA. A full 3-D magnetic field model $\mathbf{B}(x, y, z)$ is computed for each time interval and flare with a cadence of $6 \mathrm{~min}$, where the total duration of a flare is defined by the GOES flare start and end times. The size of the computation box amounts to an area with a width and length of 0.5 solar radii in the plane-of-sky, and an altitude range of 0.2 solar radius. The total number of analyzed data includes 2706 HMI images and 16,236 AIA images.

For the data analysis of this study, which is focused on scaling laws of magnetic energies dissipated during flares, we extracted from the previous studies the following observables: the mean nonpotential magnetic field strength $B_{n p}$, the mean potential field strength $B_{p}$, the mean magnetic field component $B_{\text {free }}$ associated with the free energy $E_{\text {free }}$ (or azimuthal field component $B_{\varphi}$ ), the flare area $A$, and the total magnetic energy $E_{\text {diss }}$ dissipated during the flare duration. The magnetic parameters $B_{n p}, B_{\varphi}, E_{\text {free }}, E_{\text {diss }}$ are all computed with the Vertical-Current Approximation Nonlinear Force-Free Field (VCA-NLFFF) code, as described in Paper I and Paper IX. This magnetic field extrapolation code essentially assumes vertical currents at flare locations that are associated with sub-photospheric magnetic field concentrations (e.g., sunspots and smaller magnetic features). A major advantage of this code over traditional NLFFF codes is the capability to measure the current-driven twisting of coronal magnetic field lines, based on automated tracing of coronal loops in AIA images, which this way bypasses the non-force-freeness of the photospheric field. The spatial scale $L$ is measured from the area $A=L^{2}$ of the (time-accumulated) azimuthal magnetic field, i.e., $B_{\varphi}(x, y) \geq 100 \mathrm{G}$, after correction of projection effects (Paper I). 
The multi-thermal energy $E_{t h}$ and mean electron density $n_{e}$ is obtained from a differential emission measure (DEM) analysis, using the relationship of the total emission measure $E M$ with the electron density, i.e., $E M=n_{e}^{2} V \approx$ $n_{e}^{2} L^{3}$ (Paper II). The quantities $B_{\varphi}, L, n_{e}$ are determined at the peak times of the flare emission measure, while the parameters $E_{f}$ and $E_{\text {diss }}$ are integrated over the flare duration $\tau$.

\subsection{Observed Scaling Laws}

We quantify an observed solar flare with the observables $\left[E_{p}, A_{p r o j}, l, b, D E M(T)\right]$, where $E_{p}$ is the potential magnetic energy (integrated over the volume of a computational box that encompasses a flaring active region), $A_{\text {proj }}$ is the projected 2-D area of the flaring region on the solar surface, $l$ and $b$ are the heliographic longitude and latitude, and $\operatorname{DEM}(T)$ is a differential emission measure (DEM) distribution.

First we calculate the potential field energy $E_{p}$, which can be obtained from a line-of-sight magnetogram $B_{z}(x, y)$ by means of a standard magnetic (potential) field extrapolation in the 3 -D volume $V$ of the computation box $[x, y, z]$ that encompasses a flaring active region,

$$
E_{p}=\int\left(\frac{B_{p}^{2}(x, y, z)}{8 \pi}\right) d V=\int\left(\frac{B_{p}^{2}(x, y, z)}{8 \pi}\right) d x d y d z
$$

Note that the potential field energy $E_{p}$ represents the minimum magnetic energy state, which is a lower limit of the nonpotential field (Priest 1975).

In Fig. 1a we show a linear regression fit to the free energies $E_{\text {free }}$ as a function of the potential field energies $E_{p}$, computed for 172 GOES X-class and M-class flares using the Vertical-current approximation non-linear force-free field (VCA-NLFFF) code, according to the latest version, as described in Paper IX (Fig. 8b therein). This linear regression fit yields the scaling relationship

$$
\left(\frac{E_{\text {free }}}{10^{30} \mathrm{erg}}\right)=10^{-1.73}\left(\frac{E_{p}}{10^{30} \mathrm{erg}}\right)^{1.26} .
$$

Similarly we show in Fig. $1 \mathrm{~b}$ a linear regression fit to the dissipated energies $E_{\text {diss }}$ as a function of the potential field energies $E_{p}$, as described in Paper IX (Fig. 8c therein). This linear regression fit yields the scaling relationship

$$
\left(\frac{E_{\text {diss }}}{10^{30} \mathrm{erg}}\right)=10^{-2.19}\left(\frac{E_{p}}{10^{30} \mathrm{erg}}\right)^{1.32} \text {. }
$$

We see that these two relationships are similar, in particular they have a similar power law slope of $p \approx 1.3$, while the dissipated energy contains about half of the free energy, i.e., $E_{\text {diss }} \approx E_{\text {free }} / 2$. Uncertainties of all linear regression fits are indicated in Figs. 1-2 by the mean (thick solid line) and standard deviation of the slopes (thin solid lines).

In a next step we define the scaling relationships of geometric parameters, such as the flare length scale $L$, the flare area $A$, and the flare volume $V$. We can directly observe the projected flare area $A_{\text {proj }}$ only, and estimate the true (unprojected) area $A$ by dividing with the cosine of the longitude $l$ and latitude $b$ difference to disk center,

$$
A=\frac{A_{\text {proj }}}{\cos \sqrt{l^{2}+b^{2}}} .
$$

Note that the observed flare area $A_{\text {proj }}$ is measured (in Papers I and II) from the sum of the pixel areas above some threshold of the dissipated magnetic flare energy $E_{\text {diss }}$. The time dependence of the flare area is taken into account by calculating the cumulative flare area, accumulated between the flare start and end time (Papers I and II). After we have defined the flare area $A$, we can simply define a flare length scale $L$ by

$$
L=A^{1 / 2},
$$

and a flare volume $V$ by

$$
V=A^{3 / 2} .
$$

As a next step we can investigate the relationship between the dissipated energy $E_{\text {diss }}$ and the flare volume $V$, which is shown in Fig. 1c (using the parameters determined in Paper I and shown in Fig. 14c therein). The two parameters exhibit a correlation that is fitted by the relationship,

$$
\left(\frac{V}{10^{24} \mathrm{~cm}^{3}}\right)=10^{2.34}\left(\frac{E_{\text {diss }}}{10^{30 \mathrm{erg}}}\right)^{1.17},
$$


which is almost a linear function.

Since both the flare volume $V$ and the (space-integrated) multi-thermal energy $E_{t h}$ have the same volume dependence, we expect a nearly linear relationship. There is indeed a strong correlation between these two parameters $(\mathrm{CCC}=0.91)$, as shown in Fig. 1d (or Fig. 5b in Paper II),

$$
\left(\frac{E_{t h}}{10^{30} \mathrm{erg}}\right)=10^{-1.54}\left(\frac{V}{10^{24} \mathrm{~cm}^{3}}\right)^{0.76}
$$

Another volume-dependent quantity is the emission measure $E M$, which is shown in Fig. 1e (or in Fig. 1f with reversed axes, as in Paper II, Fig. 5f therein),

$$
\left(\frac{E M}{10^{40} \mathrm{~cm}^{3}}\right)=10^{8.33}\left(\frac{E_{t h}}{10^{30} \mathrm{erg}}\right)^{0.79} .
$$

Since the differential emission measure (DEM) is generally a multi-temperature distribution, we define a weighted temperature $T_{w}$, using the integral over the function $\operatorname{DEM}(T)$,

$$
T_{w}=\frac{\int T \times D E M(T) d T}{\int D E M(T) d T}=\frac{\int T \times D E M(T) d T}{E M} .
$$

The determination of the DEM distributions $\operatorname{DEM}(T)$ using AIA/SDO data is described in Paper II and references therein. The main feature of our DEM analysis is the proper treatement of the multi-thermal distribution, rather than the commonly used iso-thermal approximation. A benchmark test with comparisons of 11 different DEM methods is described in Aschwanden et al. (2015b). In the study here we distinguish between the (temperature-integrated) emission measure $E M_{A I A}$ calculated from the 6 coronal AIA channels, and the GOES emission measure $E M_{G O E S}$, which is calculated from the iso-thermal channel ratio of the two GOES wavelength ranges 1-8 $\AA$ and 0.5-4 $\AA$.

\subsection{Scaling Law of GOES Flux}

We can now use the two parameters $\left[E M, T_{w}\right]$ to predict the GOES fluxes $F_{4}$ and $F_{8}$ in the wavelength ranges $\lambda=0.5-4 \AA$ and $1-8 \AA$. The original calibration of the GOES fluxes is given in Thomas, Starr, and Crannell (1985), and later updated by White, Schwartz, and Thomas (2005). Software capabilities to extract GOES data have been implemented in the Interactive Data Language (IDL) Solar Software (SSW) by Freeland and Handy (1998). We use the calibration of the most recent GOES spacecraft (GOES-12) given in White et al. (2005). GOES data during the SDO era were provided by GOES-15 (launched on 2010 March 4) and by GOES-16 (launched on 2016 November 19). For a given flux ratio $R$,

$$
R=F_{4} / F_{8}
$$

the GOES temperature $T_{G O E S}$ is given by a third-order polynomial (with the coefficients measured from the most recent GOES satellite (GOES-12) given in that paper),

$$
T_{\text {GOES }}=3.90+101.2 R-266.4 R^{2}+390.2 R^{3},
$$

and the parameter $b_{8}(T)$ is given by a third-order polynomial also,

$$
b_{8}(T)=-12.31+3.75 T_{G O E S}-0.1003 T_{G O E S}^{2}+0.001195 T_{G O E S}^{3},
$$

yielding the GOES emission measure $E M_{G O E S}$,

$$
E M_{G O E S}=10^{55} F_{8} / b_{8}(T) .
$$

In our application we approximate the GOES emission measure $E M_{G O E S}$ and temperature $T_{G O E S}$ with the AIAinferred emission measure $E M_{A I A}$ and emission measure-weighted temperature $T_{w}$. However, in order to allow for different temperature responses of the two instruments AIA and GOES, we define an empirical normalization factor quAnt

$$
q_{A I A}=\frac{E M_{G O E S}}{E M_{A I A}} \approx 0.54
$$


which we determine by the normalization of the predicted mean value of the GOES flux to the observed value, i.e., $F_{8}^{\text {pred }} / F_{8}^{o b s}=1.00$ (Fig. 3). We can then directly calculate the calibration parameter $b_{8}$ (Eq. 14), and predict the GOES long-wavelength flux $F_{8}$ by inverting Eq. (14),

$$
F_{8}=\frac{E M_{G O E S} b_{8}}{10^{55}}=q_{A I A} E M_{A I A} b_{8} 10^{-55} .
$$

The GOES flux is given in physical units of $\left[\mathrm{W} \mathrm{m}^{-2}\right.$, which scales with the well-known GOES flare classes as $F_{8}=10^{-6}$ $\left[\mathrm{W} \mathrm{m}{ }^{-2}\right]$ for a C1.0-class flare, $F_{8}=10^{-5}\left[\mathrm{~W} \mathrm{~m}^{-2}\right]$ for a M1.0-class flare, and $F_{8}=10^{-4}\left[\mathrm{~W} \mathrm{~m}^{-2}\right]$ for a X1.0-class flare.

In Fig. 2 we show the resulting correlations of the calculated GOES fluxes $F_{8}$ as a function of the emission measures EM (Fig. 2a), which represents the best-correlated parameter (with a cross-correlation coefficient of $\mathrm{CCC}=0.99$ ),

$$
\left(\frac{F_{8}}{\mathrm{~W} \mathrm{~m}^{-2}}\right)=10^{-14.19}\left(\frac{E M}{10^{40} \mathrm{~cm}^{3}}\right)^{1.06}
$$

There are also good correlations of the GOES flux $F_{8}$ with the thermal energy $E_{t h}$ (Fig. $2 \mathrm{~b}$ ), with a cross-correlation coeffient of $\mathrm{CCC}=0.93$,

$$
\left(\frac{F_{8}}{\mathrm{~W} \mathrm{~m}^{-2}}\right)=10^{-5.41}\left(\frac{E_{t h}}{10^{30} \mathrm{erg}}\right)^{0.84}
$$

and with the thermal flare volume $V_{t h}$, with a cross-correlation coefficient of $\mathrm{CCC}=0.72$,

$$
\left(\frac{F_{8}}{\mathrm{~W} \mathrm{~m}^{-2}}\right)=10^{-6.79}\left(\frac{V_{t h}}{10^{24} \mathrm{~cm}^{3}}\right)^{0.67} .
$$

The most important test of our scaling law relationships is the expected correlation between the observed GOES fluxes $F_{8}^{o b s}$ and the predicted values $F_{8}^{\text {pred }}$. Remember that the GOES fluxes $F_{8}$ were calculated from the emission measures $E M$ and the emission measure-weighted temperatures $T_{w}$ (Eqs. 10-15), which are obtained from the scaling laws of the emission measure $E M\left(E_{t h}\right)$ (Eq. 9), the (multi-)thermal energy $E_{t h}(V)$ (Eq. 8), the flare volume $V\left(E_{\text {diss }}\right)$ (Eq. 7), the dissipated flare energy $E_{\text {diss }}\left(E_{p}\right)$ (Eq. 3), and the potential field energy $E_{p}\left(B_{p}\right)$ (Eq. 1). Thus the final prediction of the GOES fluxes depends ultimately on the potential field $\mathbf{B}_{p}$ only. If our inferred scaling laws are correct, we would expect a close correlation between the observed $\left(F_{8}^{o b s}\right)$ and the predicted GOES fluxes $\left(F_{8}^{\text {pred }}\right)$. The analytical derivation of this GOES scaling law is given in Appendix A. We show a cross-correlation plot of these two quantities in Fig. (2d), which reveals a reasonably good cross-correlation coefficient of CCC $=0.71$,

$$
\left(\frac{F_{8}^{\text {pred }}}{\mathrm{W} \mathrm{m}^{-2}}\right)=10^{0.80}\left(\frac{F_{8}^{o b s}}{\mathrm{~W} \mathrm{~m}^{-2}}\right)^{1.18}
$$

This test establishes our claim that we can predict the GOES class of a flare, based on the potential field alone. Since the temporal evolution of the potential field is generally relatively slow, it provides a robust prediction for the flare magnitude (i.e., GOES class).

In Fig. 3 we show the ratios $q_{a c c}$ of the predicted $F_{8}^{\text {pred }}$ to the observed GOES values $F_{8}^{o b s}$, which exhibit a Gaussian-like distribution with a mean and standard deviation of $q_{a c c}=1.8 \pm 0.6$, for the 172 flare events investigated in this study. Normalizing the AIA-inferred multi-thermal emission measure $E M_{A I A}$ to the GOES-inferred isothermal emission measure $E M_{G O E S}$, we find a normalization factor of $q_{A I A}=E M_{G O E S} / E M_{A I A}=0.54$, which has a mean and standard deviation of $q_{A I A}=1.00 \pm 0.40$ (Fig. 3) and implies that the accuracy of the GOES scaling laws is $\approx \pm 40 \%$.

\subsection{Simplified GOES Class Estimates}

Our goal is the prediction of the GOES (1-8 $\AA$ ) flux $F_{8}$ for each flare, based on information on the potential field energy $E_{p}$. A direct scaling relationship between these two parameters $F_{8}$ and $E_{p}$ can be obtained by combining all Eqs. (1-15),

$$
F_{8}=q_{A I A} b_{8}\left(T_{w}\right) 10^{-8}\left(\frac{E_{p}}{10^{30} \mathrm{erg}}\right)^{0.92}\left[\mathrm{~W} \mathrm{~m}^{-2}\right]
$$


where the detailed analytical derivation is given in Appendix A. Since the emission measure-weighted temperature in our sample of $172 \mathrm{X}$ and M-class flare events is relatively narrow, varying only by a factor of $\approx 20 \%$,

$$
T_{w} \approx 25.5 \pm 5.6[\mathrm{MK}]
$$

we obtain a mean calibration factor $b_{8}\left(T_{w}\right)$ of,

$$
b_{8}\left(T_{w}\right) \approx 38,
$$

which together with the normalization factor $q_{A I A}=0.54$ yields then the simple scaling law.

$$
F_{8} \approx 2.05 \times 10^{-7}\left(\frac{E_{p}}{10^{30} \mathrm{erg}}\right)^{0.92} \quad\left[\mathrm{~W} \mathrm{~m}^{-2}\right] .
$$

This simplified scaling law allows quick estimates of the GOES class: Total (potential field) energies of $E_{p} \approx 5,50$, and $500 \times 10^{30} \mathrm{erg}$ are required for flare magnitudes of GOES class C1.0, M1.0, and X1.0. We will use the simplified scaling law of Eq. (24) for flare forecasting in the following.

\subsection{Daily Forecasting of GOES Flare Magnitude}

In this section we generate a new data set that produces a time series of magnetic potential field energies $E_{p}(t)$, using the Vertical-Current Approximation Non-Linear Force-Free Field (VCA-NLFFF) code. Detailed descriptions of the magnetic field code are given in Aschwanden et al. (2014b, 2016). We use observations of active regions from HMI/SDO and AIA/SDO during the month of 2011 February 1-28, which we analyze with a cadence of $\Delta t=24$ hours. The Sun is very active during this month, producing one X2.2 GOES class flare and 13 M-class flares (Table 1). We use the NOAA flare catalog (hesperia.gsfc.nasa.gov) to identify the heliographic coordinates of the active regions. There are one, none, or multiple active regions present every day, for which we have to calculate magnetic field extrapolations separately, and have to add them up in order to gather all significant magnetic energies on the visible solar disk. For the month of February 2011 we find a total of 23 days (out of the 28 days) with active regions present, encompassing a total of 53 active regions, according to the NOAA data catalog.

Our main goal is to test whether our anticipated algorithm is able to predict upper limits of the GOES 1-8 $\AA$ flux $F_{8}(t)$ based on the potential magnetic energies only (i.e., using the scaling law $F_{8}(t) \propto E_{p}(t)^{0.92}$ (Eq. 24). First we show the obtained time profiles of the potential energy $E_{p}(t)$ (Fig. 4a), the nonpotential energy $E_{n p}(t)$ (Fig. $4 \mathrm{~b}$ ), and the free energy $E_{\text {free }}(t)$ (Fig. 4c). For comparison we show also the results of an earlier version of the VCA-NLFFF code (noisy time profiles in Figs. 4a and 4b) for the time segment of 2011 Feburary 12-17 (Aschwanden et al. 2016). The time evolution of the potential and nonpotential energy is in good agreement between the old and new version of the VCA-NLFFF code (Figs. $4 \mathrm{a}$ and $4 \mathrm{~b}$ ), while the free energy exhibits slight differences, caused by the fact that the free energy is a small difference of two large quantities, i.e., $E_{f r e e}=E_{n p}-E_{p}$, and thus has a larger relative uncertainty.

We show the GOES 1-8 A flux time profile is Fig. 4d (grey area), which contains $14 \mathrm{M}$ and X-class flare events during the investigated time window of 28 days. First we consider the "now-cast" case, where the predicted time is identical to the observed time. We take the potential field energy $E_{p}(t)$ and calculate the upper limit of the GOES flux $F_{8}(t)$ with the scaling law given in Eq. $(24)$, i.e., $F_{8}(t) \propto E_{p}(t)^{0.92}$ and overplot it on the actually observed GOES 1-8 A flux in Fig. 4d (grey area), which demonstrates that the so derived GOES fluxes indeed represent reliable upper limits as a function of time. The uncertainty of the empirical scaling law amounts to $q_{a c c}=F_{8}^{\text {pred }} / F_{8}^{o b s}=1.0 \pm 0.4$ (Fig. 3). This means that we expect a probability of $67 \%$ for one standard deviation $(\sigma= \pm 0.4)$.

Next we consider the "flare prediction" case (of the upper limit of the GOES flux $F_{8}^{\text {pred }}(t)$ ), for a time interval of $\Delta t=24$ hours ahead of the observing time, which is shown in Fig. 4e (thick solid line). The predicted value $E_{p}(t)$ is computed by linear extrapolation from the past two time intervals, i.e., $E_{p}(t)=2 * E_{p}(t-\Delta t)-E_{p}(t-2 \Delta t)$, and apply then the empirical scaling law $F_{8}(t) \propto E_{p}(t)^{0.92}$. To avoid unphysical negative values in the extrapolated potential energy $E_{p}(t)$, we extrapolate the logarithmic values.

The four largest flares (see Table 1) occur on 2011 February 13, 15, 18, and 24, with GOES fluxes of M6.6, M2.2, M6.6, and M3.5 (indicated in Fig. 4e), for which we predict upper limits of M7.3, X1.4, M7.5, and M8.3, corresponding to flux ratios of $q_{8}=F_{8}^{o b s} / F_{8}^{\text {pred }}=0.9,1.5,0.9$, and 0.4 , which is approximately consistent with our expectation of $q_{a c c}=1.0 \pm 0.4$ (Fig. 3). Both the predicted upper limit of the GOES flux (Fig. 4e, thick curve) and the limit of the now-cast case (Fig. 4e, dashed curve), are shown together in Fig. (4e), so that the uncertainty due to the temporal extrapolation can be seen. The comparison corroborates our assumption that the potential energy $E_{p}(t)$ is relatively slowly-varying on time scales of a day. 


\section{DISCUSSION}

\subsection{Physical Parameters Relevant for Flare Prediction}

A magnetic potential field represents the minimum energy state (Priest 1975), from which no energy can be dissipated to produce a solar flare. Only the free energy $E_{f r e e}=E_{n p}-E_{p}$, which is the difference between the non-potential field and the potential field energy, can be dissipated in flares, which constrains the choice of valid physical parameters in flare models. A selection of 25 physical parameters that can be measured in active regions has been presented by Bobra and Couvidat (2015), and a subset of 18 parameters thereof in Bobra and Ilionidis (2016), drawn from the Space-weather HMI Active Region Patches (SHARP) project. For flare predictions, however, only those parameters are relevant that are directly sensitive to the free energy $E_{\text {free }}>0$, or to the underlying electric current density $\nabla \times \mathbf{B}=4 \pi \mathbf{j}=\alpha(\mathbf{r}) \mathbf{B}$. In the study of Bobra and Couvidat (2015) we identify 17 relevant physical parameters, which quantify the free energy $\left(\rho_{\text {tot }}, \bar{\rho}\right)$, the vertical currents $\left(J_{z, \text { total }}, J_{z, \text { sum }}, \overline{J_{z}}\right)$, the helical twist angle $\left(\alpha_{\text {total }}\right)$, the shear angle $(\bar{\Gamma})$, the helicity $\left(H_{z, t o t a l}, H_{a b s}, \overline{H_{c}}\right)$, and the Lorentz force $\left(F, F_{z}, F_{y}, F_{x}, \delta F_{x}, \delta F_{y}, v \delta F_{z}\right)$. The Lorentz force depends on the free energy component $B_{\text {free }}$ only, i.e., $\mathbf{F} \propto \mathbf{j} \times \mathbf{B}_{n p}=\mathbf{j} \times \mathbf{B}_{p}+\mathbf{j} \times \mathbf{B}_{\text {free }}=\mathbf{j} \times \mathbf{B}_{\text {free }}$, since there is no current in the potential field. The other forthcoming parameters are sensitive to the nonpotential magnetic field strength $\mathbf{B}_{n p}=\left[B_{x}, B_{y}, B_{z}\right]$, rather than to the magnetic field component associated with the free energy, i.e., $\mathbf{B}_{\text {free }}=\mathbf{B}_{n p}-\mathbf{B}_{p}$. The remaining parameters, such as the the magnetic flux $\left(\Phi, \Phi_{R}\right)$, the flare area $\left(A, A_{45}\right)$, the field divergence $\left(\overline{\nabla B_{t o t}}, \overline{\nabla B_{z}}, \nabla B_{h}\right)$, or the inclination angle $\bar{\gamma}$, are thus not directly relevant for flare predictions, although they may be useful in characterizing (non-flaring) active regions.

\subsection{Flare Prediction Algorithms}

Early flare prediction algorithms were based on empirical (morphological) parameters rather than physical models. For instance, Gallagher et al. (2002) developed a flare prediction system which estimates the probability for each active region to produce $\mathrm{C}-$, $\mathrm{M}$-, or X-class flares based on sunspot classification.

With increasing sophistication, a trend from morphological parameters to physical parameters took place, mostly measured from line-of-sight magnetograms. For instance, a method of partitioning the magnetic flux in a magnetogram and deriving the magnetic connectivity has been used in forecasting of major flares (Barnes et al. 2005; Georgoulis and Rust 2007). The lower moments of the field gradients, the kurtosis of the vertical current density, the magnetic twist, the current helicity density, and the magnetic shear angle has been used in another series of studies (Leka and Barnes 2003, 2007; Barnes et al. 2007; Barnes and Leka 2008). It was noted that parameters that depend on the size of a flare are better correlated with the flaring behavior than parameters that do not depend on the system size (Welsch et al. 2009), which is consistent with our definition of the behavior of the free energy, which is spatially integrated over the entire flaring active region and thus depends on the system size.

Ultimately we aim to find physical flare models that can predict the onset of a flare instability, if solar flares are produced by a deterministic process. However, many studies show that flaring appears to be a stochastic process, which cannot predict individual flares, rather than a deterministic process. Neverthelss, flare forecasting has been approached by machine-learning algorithms (e.g., Bobra and Couvidat 2015; Bobra and Ilionidis 2016), which optimize prediction skills, regardless of whether the flare process is stochastic or deterministic. The algorithm of Bobra and Couvidat (2015) is based on binary classifiers, where an active region belongs to a positive class if it produces one or more flares within a given time interval, and conversely, an active region belongs to a negative class if it does not produce a flare in the same time interval. This is slightly different from our method with one qualifier, where only an upper limit of a flare magnitude is predicted within a time window in the near future. Thus, we do not predict the actual time when a flare occurs, but only an upper limit in case a flare occurs. This relieves also the class-imbalance problem (Bobra and Couvidat 2015). In our method we can identify two types of uncertainties: (i) The error of the theoretical scaling law (which can be measured from the difference of theoretical and observational values at now-cast time $t=t_{\text {now }}$ ), and (ii) the time extrapolation error (which can be measured from the difference in the prediction time window, at $\left(t>t_{\text {now }}\right)$. From the 25 physical parameters tested in Bobra and Couvidat (2015), we identify 10 relevant physical parameters that are sensitive to the free energy, the vertical current, the helical twist angle, the shear angle, or helicity, in agreement with our physical model of the Vertical Current Approximation (VCA) NLFFF code. The underlying physical model is essentially a force-free solution of helically twisted magnetic field lines, which are generated by vertical currents emanating above (sub-photospheric) magnetic flux concentrations. If this physical model is correct, we expect that these physical parameters have a relatively high prediction score, which is indeed largely the case. However, the observables in the study of Bobra and Couvidat (2015) are based on photospheric magnetograms, which 
is not force-free (Metcalf et al. 1996), while the magnetic parameters in this study here are derived with the VCANLFFF code by fitting of coronal loops, which are thought to be force-free (with a plasma- $\beta$-parameter $\beta \ll 1$ ), and thus are expected to provide a more realistic magnetic field model.

\subsection{GOES Fluxes and Flare Magnitude}

The GOES 1-8 A flux is often used to characterize the flare magnitude, mostly because of the convenience of the permanent availability of GOES observations (since 1974). It is therefore important to quantify how well the GOES flux correlates with other flare magnitude indicators. In this study we include large flares (of X and M-class) only, which are the most relevant events to derive upper limits of the GOES flux, while smaller flares (of C and B class) are neglected here, since we are not interested in representative distribution functions, which would exhibit power-laws (e.g., Aschwanden and Freeland 2012). The GOES flux appears to correlate with the upper limit of the absolute total flux near the strong-field, high-gradient polarity inversion lines (Schrijver 2007; 2009). A strong correlation has been established between the GOES flux and the thermal energy (Reep et al. 2013, 2020), or between the temperature as well as the emission measure of the thermal plasma and the GOES flux (Warmuth et al. 2016a; 2016b). Even for cool (small) flares of GOES class B5 to C2, the emission measure was found to be correlated with the GOES flux (Phillips and Feldman 1995). Correlations between the GOES flux and the AIA/SDO or EUVI/STEREO flux have been found for occulted flares (Nitta et al. 2013). However, using large statistics of 50,000 solar flares observed during three solar cycles, the exact values of GOES peak temperatures and emission measures were found to depend on the background subtraction method (Ryan et al. 2012), originally pointed out by Bornman (1990). However, for large (M and X-class) flares, as used here, the background correction is negligible.

We have to mention the caveat that the calculation of the GOES emission measure and temperature from an inversion of the two-channel GOES fluxes (1-8 $\AA$ and 0.5-4 $\AA$ ), is based on the isothermal assumption (Thomas et al. 1985; White et al. 2005), and thus is generally not compatible when comparing different instruments, such as AIA, GOES, and RHESSI (Ryan et al. 2014). Here we find an empirical normalization factor for the emission measure obtained from GOES and AIA (i.e., $q_{A I A}=E M_{G O E S} / E M_{A I A} \approx 0.54$ (Eq. 15).

In summary, while all previously published studies agree that the emission measure $E M$ is highly correlated with the GOES flux $F_{8}$ (Eq. 17), we find that the underlying cross-correlation coefficient has actually the highest value $(\mathrm{CCC}=0.99)$ among all investigated correlations, and thus both the emission measure as well as the GOES class (flux) are equally good measures of the flare magnitude, analogously to the Gutenberg-Richter scale (or magnitude) of earthquakes.

\section{CONCLUSIONS}

Solar flare prediction is a highly desirable goal, as the host of hundreds of scientific publications over the last decade reveals. However, the terms "prediction" or "forecasting" have different meanings to researchers. We can distinguish between at least two different types of predictions: (i) the deterministic approach, which aims to predict the occurrence time and size of individuial events, and (ii) the stochastic approach, which predicts statistical distributions only, without any temporal evolution of individual events. The deterministic approach is of course the more challenging task, because it requires an accurate and realistic physical model, while the stochastic approach just requires statistics of large samples. Extreme events are often produced by nonlinear physical mechanisms, which have the tendency to form "fat tails" in their statistical distributions in form of power-law or log-normal functions. A popular new type of methods is "machine-learning ", which is able to learn and improve predictions by optimizing parameter combinations from an initial "training data set". However, successful machine-learning can only be accomplished if the most relevant phyiscial parameters are included in the training data set, which is essentially not available as long as we do not have knowledge of the correct physical mechanism. In this study we explore empirical scaling laws that quantify realistic parameter distributions and significant correlations of physical parameters in solar flares, which can be extrapolated into the near future and predict reliable upper limits of flare magnitudes (in terms of GOES fluxes). Here the main conclusions:

1. The success of any flare prediction algorithm rests in the selection of relevant physical parameters. Since the (magnetic) potential field represents the minimum state of energy, only the free energy $E_{\text {free }}=E_{n p}-E_{p}$ can be dissipated in flares, which requires knowledge of the three magnetic field components $\mathbf{B}_{p}, \mathbf{B}_{n p}$, and $\mathbf{B}_{f r e e}$. Scaling laws that do not take into account the free energy explicitly may be unphysical. 
2. Another inconsistency that appeared in the extrapolation of both the potential or the non-potential magnetic field is the neglect of the photospheric non-force-freeness, a common assumption in traditional NLFFF codes. Here we bypass the non-force-freeness of the photosphere by fitting coronal loops (which are supposed to be forcefree in the corona due to the low plasma- $\beta$ parameter), using the vertical current approximation (VCA-NLFFF) code. This may affect the magnetic field parameters and their correlations in flare scaling laws.

3. Two-parameter correlations (Fig. 1) constitute a subset of simplified or approximative scaling laws, which we explore in this study. The best correlations among physical flare parameters are found for the free energy and potential field energy, $E_{\text {free }} \propto E_{p}^{1.26}$; the dissipated energy, $E_{\text {diss }} \propto E_{p}^{1.32}$; the magnetic flare volume, $V \propto E_{\text {diss }}^{1.17}$; the (multi-)thermal energy, $E_{t h} \propto V_{t h}^{0.76}$; the emission measure, $E M \propto E_{t h}^{0.79}$, and the GOES flux, $F_{8} \propto E_{p}^{0.92}$. The fact that these parameters exhibit tight correlations makes them highly relevant for flare scaling laws.

4. In the opposite, uncorrelated parameters do not reveal scaling laws. This is evident, for instance, from scatterplots of the electron density $n_{e}$ or the electron temperature $T_{e}$ with other flare parameters, if we restrict to $\mathrm{M}$ and $\mathrm{X}$-class flares only. However, both the electron density and electron temperature are correlated with the GOES flux if one includes C and B-class flares (Feldman et al 1996). Here we restrict ourselves to large flares of X and M-class for the prediction of upper limits of GOES fluxes, where the emission measure-weighted temperature $\left(T_{w}=25 \pm 5 \mathrm{Mm}\right)$ has a standard deviation of $\pm 20 \%$ only. The electron density $n_{e}$ has a similar small spread of less than an order of magnitude (e.g., Aschwanden 2020), while all volume-dependent flare parameters exhibit a much larger spread (some 2-3 order of magnitudes for X- and M-class flares), and thus dominate the twoparameter correlations.

5. Among the parameters that correlate best with the GOES flux (Fig. 2), we find the emission measure, $F_{8} \propto$ $E M^{1.06}$; the thermal energy, $F_{8} \propto E_{t h}^{0.84}$; and the thermal flare volume, $F_{8} \propto V_{t h}^{0.66}$. Comparing the observed GOES-class values $\left(F_{8}^{o b s}\right)$ with the theoretically predicted values $\left(F_{8}^{\text {pred }}\right)$ based on the two-parameter scaling laws we find a satisfactory agreement of $q_{a c c}=F_{8}^{\text {pred }} / F_{8}^{o b s}=1.00 \pm 0.40$, after normalization of the fluxes. Thus, the accuracy of predicted GOES-class magnitudes is of order $\pm 40 \%$ (Fig. 3).

6. Our flare prediction method yields upper limits of the GOES flux $F_{8}^{\text {theo }}(t)$, either instantaneously for now-casting (Fig. 4d), or during time intervals of at least $\Delta t \leq 24 \mathrm{hrs}$ (Fig. 4e) for fore-casting. The chief assumption is the slow time variability of the potential field energy $E_{p}(t)$ on time scales of $\leq 24$ hrs, which allows the temporal extrapolation of the scaling law $F_{8}(t) \propto E_{p}(t)^{0.92}$ (Fig. 4). We tested our fore-casting method over a time interval of 28 days and validated our claim that this method yields reliable upper limits of the GOES flux. Future applications to longer time intervals with larger flare statistics will allow us also to verify the statistical probabilities of the most extreme events, which exceed the predicted upper limits, also known as "Dragon-King" events (Sornette 2009; Sornette and Ouillon 2012; Aschwanden 2019b).

7. We have to be aware that two-parameter correlations represent flare scaling laws with a minimal number of parameters, which can be refined for specific flare models (such as the Sweet-Parker or Petschek model). For instance, a Petschek-type flare model can be quantified with 8 physical parameters for the dissipated flare energy, $E_{M R} \propto B_{\text {free }}^{2} B_{n p} L n_{e}^{1 / 2} \lambda M_{A} \tau_{\text {flare }}$, with $E_{M R}$ the dissipated energy, $B_{\text {free }}$ the magnetic field component associated with the free energy, $B_{n p}$ the non-potential field strength, $L$ the flare length scale, $n_{e}$ the electron density, $\lambda$ the electron density scale height, $M_{A}$ the Mach number of the magnetic reconnection outflow, and $\tau_{\text {flare }}$ the flare duration (Aschwanden 2020).

The aim of this study is the distillation of the most relevant physical parameters that play a role in flare prediction methods. Once we manage this task, deterministic as well as stochastic flare prediction becomes a promising option, at least over a time interval that exhibits coherent magnetic field evolution. Future work may test the reliability of predicting upper limits for the GOES flux over the entire 10-year interval of the SDO mission. Furthermore, various machine-learning methods applied to the most relevant physical parameters may optimize realistic scaling laws.

Acknowledgements: We thank the anonymous referee and the statistical editor for helpful comments. We acknowledge software support by Samuel Freeland, Greg Slater, and Mark Noga. Part of the work was supported 
by NASA contract NNG 04EA00C of the SDO/AIA instrument and the NASA STEREO mission under NRL contract N00173-02-C-2035.

\section{APPENDIX A : DERIVATION OF SCALING LAWS FOR GOES FLUX}

The scaling laws between the magnetic potential field energy $E_{p}$, the dissipated energy in flares $E_{\text {diss }}$, the thermal flare volume $V_{t h}$, the (multi-)thermal flare energy $E_{t h}$, and the emission measure $E M$ are specified in Eqs. $(9,8,7,3)$,

$$
\begin{aligned}
& \left(\frac{E M}{10^{40} \mathrm{~cm}^{3}}\right)=10^{a_{0}}\left(\frac{E_{t h}}{10^{30} \mathrm{erg}}\right)^{a_{1}}, \\
& \left(\frac{E_{t h}}{10^{30} \mathrm{erg}}\right)=10^{b_{0}}\left(\frac{V_{t h}}{10^{24} \mathrm{~cm}^{3}}\right)^{b_{1}}, \\
& \left(\frac{V_{t h}}{10^{24} \mathrm{~cm}^{3}}\right)=10^{c_{0}}\left(\frac{E_{\text {diss }}}{10^{30} \mathrm{erg}}\right)^{c_{1}}, \\
& \left(\frac{E_{\text {diss }}}{10^{30} \mathrm{erg}}\right)=10^{d_{0}}\left(\frac{E_{p}}{10^{30} \mathrm{erg}}\right)^{d_{1}},
\end{aligned}
$$

with the coefficients $a_{0}=8.33, a_{1}=0.79, b_{0}=-1.54, b_{1}=0.76, c_{0}=2.34, c_{1}=1.17, d_{0}=-2.19, d_{1}=1.32$, as determined from the linear regression fits shown in Fig. 1. The scaling law of the GOES flux $F_{8}$ as a function of the emission measure $E M$ is according to Eq. (16),

$$
F_{8}(E M)=f_{0} E M, \quad f_{0}=q_{A I A} b_{8}\left(T_{w}\right) 10^{-55} .
$$

The second relationship of the GOES flux $F_{8}\left(E_{t h}\right)$ as a function of the thermal energy $E_{t h}$ is obtained by inserting Eq. (A1) into Eq. (A5),

$$
F_{8}\left(E_{t h}\right)=f_{0} 10^{40+a_{0}}\left(\frac{E_{t h}}{10^{30} \mathrm{erg}}\right)^{a_{1}} .
$$

The third relationship of the GOES flux $F_{8}\left(V_{t h}\right)$ as a function of the thermal flare volume $V_{t h}$ is obtained by inserting Eq. (A2) into Eq. (A6),

$$
F_{8}\left(V_{t h}\right)=f_{0} 10^{40+a_{0}+b_{0} a_{1}}\left(\frac{V_{t h}}{10^{24} \mathrm{erg}}\right)^{a_{1} b_{1}} .
$$

The fourth relationship of the GOES flux $F_{8}\left(E_{\text {diss }}\right)$ as a function of the magnetic dissipated energy $E_{\text {diss }}$ is obtained by inserting Eq. (A3) into Eq. (A7),

$$
F_{8}\left(E_{\text {diss }}\right)=f_{0} 10^{40+a_{0}+b_{0} a_{1}+c_{0} a_{1} b_{1}}\left(\frac{E_{\text {diss }}}{10^{30} \mathrm{erg}}\right)^{a_{1} b_{1} c_{1}} .
$$

The fifth relationship of the GOES flux $F_{8}\left(E_{p}\right)$ as a function of the magnetic potential energy $E_{p}$ is obtained by inserting Eq. (A4) into Eq. (A8),

$$
F_{8}\left(E_{p}\right)=f_{0} 10^{40+a_{0}+b_{0} a_{1}+c_{0} a_{1} b_{1}+d_{0} a_{1} b_{1} c_{1}}\left(\frac{E_{p}}{10^{30} \mathrm{erg}}\right)^{a_{1} b_{1} c_{1} d_{1}} .
$$

which yields to the numerical values given in Eqs. (21) and (24). This scaling law stated in Eq. (24) is most useful for flare prediction in terms of the GOES flux $F_{8}$ as a function of the magnetic potential field energy $E_{p}$. 


\section{REFERENCES}

Aschwanden, M.J. and Freeland, S.L. 2012, A\&A 754, 112

Aschwanden, M.J., Xu, Y., and Jing, J. 2014a, ApJ 797:50 (Paper I)

Aschwanden, M.J., Sun, X., and Liu, W. 2014b, ApJ 785:34

Aschwanden, M.J., Boerner, P., Ryan, D., Caspi, A., McTiernan, J.M., and Warren, H.P. 2015a, ApJ 802:53 (Paper II)

Aschwanden, M.J., Boerner, P., Caspi, A., McTiernan, J.M., Ryan, D., and Warren, H. 2015b, SoPh 290, 2733

Aschwanden, M.J. 2016, ApJSS 224, 225

Aschwanden, M.J. 2019a, ApJ 885:49 (Paper IX)

Aschwanden, M.J. 2019b, ApJ 880:105

Aschwanden, M.J. 2020, ApJ (in press), Global Energetics of Solar Flares: Petschek Reconnection Rate and Alfvén Mach Number of Magnetic Reconnection Outflows

Barnes, G., Longcope, D.W., and Leka, K.D. 2005, ApJ 629, 561

Barnes, G., Leka, K.D., Schumer, E.A., and Della-Rose, D.J. 2007, Space Weather 5,9, CiteID S09002

Barnes, G. and Leka, K.D. 2008, ApJ 688, L107

Barnes, G., Leka, K.D., Schrijver, C.J., et al. 2016, ApJ 829, 89

Bobra, M.G. and Couvidat, S. 2015, ApJ 798:135

Bobra, M.G. and Ilionidis, S. 2016, ApJ 821:127

Bornmann P.L. 1990, ApJ 356, 733

Cui, Y., Li, R., Zhang, L. et al. 2006, SoPh 237, 45

Falconer, D.A., Moore, R.L., and Gary, G.A. 2003, JGR 108, A10, 1380

Falconer, D.A., Abdulnasser, F., Khazanov, I. et al. 2011, Space Weather 9/4, S04003

Falconer, D.A., Moore, R.L., Barghouty, A.F. et al. 2012, ApJ 757, 32

Feldman, U., Doschek, G.A., Behring, W.E., and Phillips, K.J.H. 1996, ApJ 460, 1034

Fleck, B., Domingo, V., and Poland, A. (eds.) 1995, SoPh 162

Freeland, S.L. and Handy, B.N. 1998, SoPh 182, 497

Gallagher, P.T., Moon, Y.J., and Wang, H. 2002, SoPh 209, 171

Georgoulis, M.K. and Rust, D.M. 2007, ApJ 661, L109.

Georgoulis, M.K. 2012, SoPh 276, 161

Jonas, E., Bobra, M., Shankar, V. et al. 2018, SoPh 293, 48

Leka, K.D. and Barnes G. 2007, ApJ 656, 1173

Leka, K.D. and Barnes, G, 2003, ApJ 595, 1277

Lemen, J.R., Title, A.M., Akin, D.J., et al. 2012, SoPh 275, 17

Lemen, J.R., Duncan, D., Edwards, C., et al. 2004, SPIE 5171, 65

Metcalf, T.R., Litao, J., McClymont, A.N., and Canfield, R.C., 1995, ApJ 439, 474

Nishizuka, N., Sugiura, K., Kubo, Y., Den, M., Watari, S., and Ishii, M. 2017, ApJ 835, 156

Nitta, N.V., Aschwanden, M.J., Boerner, P.F., Freeland, S.L., Lemen, J.R., and Wuelser, J.P. 2013, SoPh 288, 241

Pesnell, W.D., Thompson, B.J., and Chamberlin, P.C. 2012, SoPh 275, 3

Phillips, K.J.H. and Feldman, U. 1995, A\&A 304, 563

Priest, E.R. 1975, SoPh 43, 177

Reep, J.W., Bradshaw, S.J., and McAteer, R.T.J. 2013, ApJ 778:76

Reep, J.W., Antolin, P., and Bradshaw, S.J.et al. 2020, ApJ 890, Id. 100

Ryan, D.F., Gallather, P.T., Dennis, B.R., Tolbert, A.K., Schwartz, R.A., and Young, C.A. 2012, ApJSS 202, id. 11

Ryan, D.F., O'Flannagaink A.M., Aschwanden, M.J., and Gallagher P.T. 2014, SoPh 289, 2547

Scherrer, P.H., Bogart, R.S., Bush, R.I. et al. 1995, SoPh 162, 129

Scherrer P.H., Schou, J., Bush, R.J. 2012, SoPh 275, 207

Schrijver, C.J., Sandman, A.W., Aschwanden, M.J., and DeRosa, M.L. 2004, ApJ 615, 512

Schrijver, C.J. 2007, ApJ 655, L117

Schrijver, C.J. 2009, Adv. Space Res. 43, 739

Sornette, D. 2009, J. Terraspace Science and Engeneering, 2, 1

Sornette, D. and Ouillon, G. 2012, EPJST, 205, 1

Tiwari, S.K., Venkatakrishnan, P., and Gosein, S. et al. 2010, ApJ 721, 622. 
Thomas, R.J., Starr, R., and Crannell, C.J. 1985, SoPh 95, 323

Warmuth, A. and Mann, G. 2016a, A\&A 588, A115

Warmuth, A. and Mann, G. 2016b, A\&A 588, A116

Welsch, B.T., Li, Y., Schuck, P.W., and Fisher, G.H. 2009, ApJ 705, 821

Wheatland, M.S. 2005, Space Weather 3/7, S07003

White, S.M., Thomas, R.J., and Schwartz, R.A. 2005, SoPh 227, 231

Wiegelmann, T., Inhester, B., and Sakurai, T. 2006, SoPh 223, 215 
Table 1. Observed and predicted upper limits of the GOES 1-8 A flux during the month of 2011 February 1-28, containing a total of $14 \mathrm{X}$ and M-class flares.

\begin{tabular}{|c|c|c|c|c|c|}
\hline Date Time & $\begin{array}{l}\text { Heliographic } \\
\text { position }\end{array}$ & NOAA & $\begin{array}{l}\text { GOES } \\
\text { flux } \\
F_{8}^{o b s} \\
{\left[\mathrm{~W} \mathrm{~m}^{-2}\right]}\end{array}$ & $\begin{array}{l}\text { Predicted } \\
\text { upper limit } \\
F_{8}^{\text {pred }} \\
{\left[\mathrm{W} \mathrm{m} \mathrm{m}^{-2}\right]} \\
\end{array}$ & $\begin{array}{l}\text { Ratio } \\
F_{8}^{o b s} / F_{8}^{\text {pred }}\end{array}$ \\
\hline 2011-02-09 01:23:00 & N16W70 & 11153 & M1.9 & M1.0 & 1.9 \\
\hline 2011-02-13 17:28:00 & S21E04 & 11158 & M6.6 & M7.3 & 0.9 \\
\hline 2011-02-14 17:20:00 & S20W07 & 11158 & $\mathrm{M} 2.2$ & $\mathrm{X} 1.1$ & 0.2 \\
\hline 2011-02-15 01:44:00 & S21W12 & 11158 & $\mathrm{X} 2.2$ & $\mathrm{X} 1.4$ & 1.5 \\
\hline 2011-02-16 01:32:00 & S22W27 & 11158 & M1.0 & M7.7 & 0.1 \\
\hline 2011-02-16 07:35:00 & S23W30 & 11161 & M1.1 & M6.7 & 0.2 \\
\hline 2011-02-16 14:19:00 & S23W33 & 11158 & M1.6 & M5.8 & 0.3 \\
\hline 2011-02-18 09:55:00 & S21W55 & 11158 & M6.6 & M7.5 & 0.9 \\
\hline 2011-02-18 10:23:00 & N17E07 & 11162 & M1.0 & M7.4 & 0.1 \\
\hline 2011-02-18 12:59:00 & S20W70 & 11158 & M1.4 & M7.2 & 0.2 \\
\hline 2011-02-18 14:00:00 & N17E04 & 11162 & M1.0 & M7.1 & 0.1 \\
\hline 2011-02-18 20:56:00 & N15E00 & 11162 & M1.3 & M6.4 & 0.2 \\
\hline 2011-02-24 07:23:00 & N14E87 & 11163 & M3.5 & M8.3 & 0.4 \\
\hline 2011-02-28 12:38:00 & N22E35 & 11164 & M1.1 & M1.0 & 1.1 \\
\hline
\end{tabular}



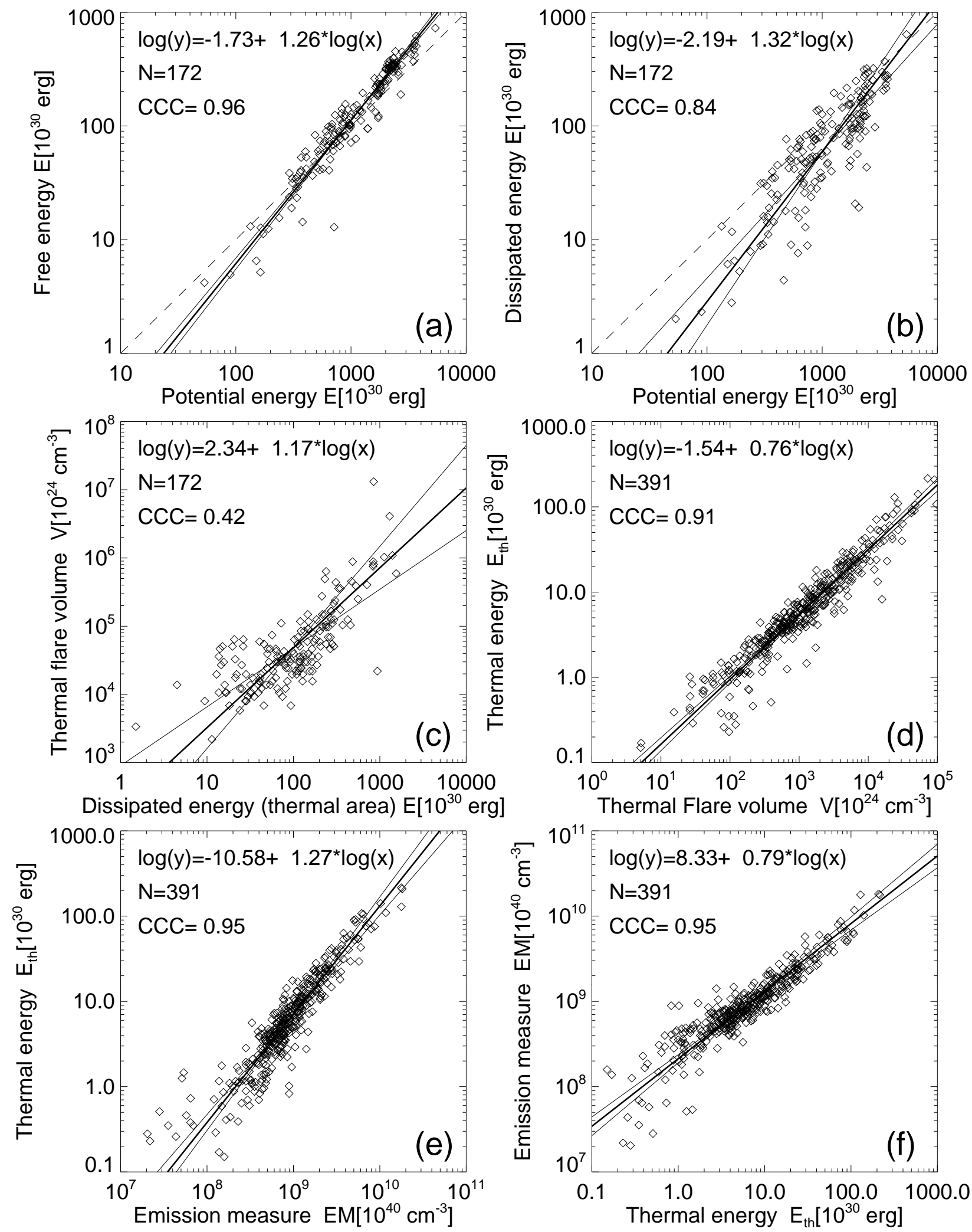

Figure 1. Correlations between the observed values (diamonds) as a function of the potential energy are shown for the free energy (a), the dissipated energy (b), the flare volume (c), the thermal energy (d), and the emission measure (e,f). Linear regressions are shown for the logarithmic values, with the mean (thick solid lines) and standard deviation (thin solid lines), and equivalence (dashed lines) indicated. 

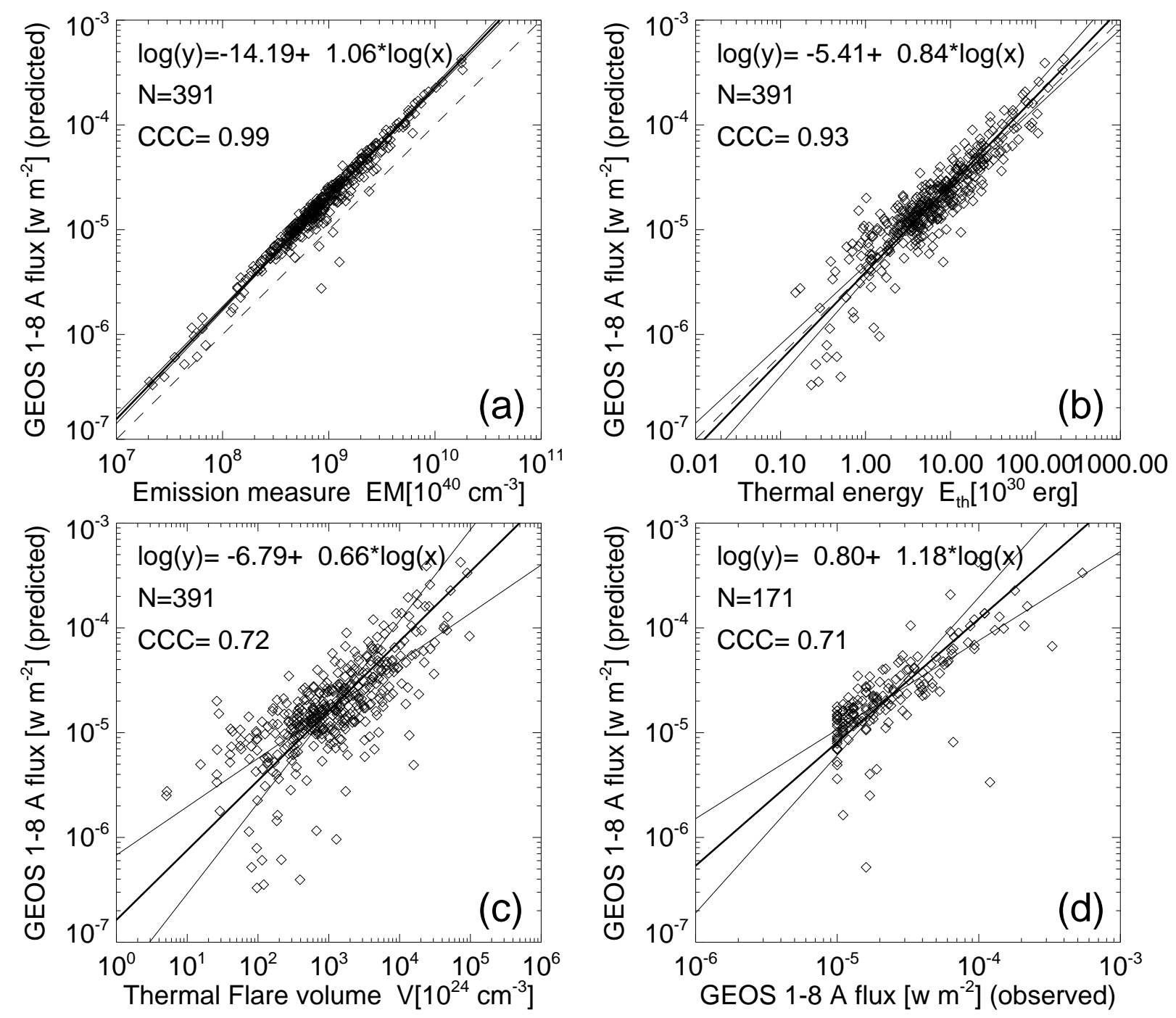

Figure 2. Correlations between the calculated GOES class and the emission measure $E M$ (a), the (multi-)thermal energy $E_{t h}$ (b), the flare volume $V$, and the observed GOES class (d). Linear regressions are shown for the logarithmic values, with the means (thick solid lines) and standard deviations (thin solid lines), and equivalence (dashed lines) indicated. 


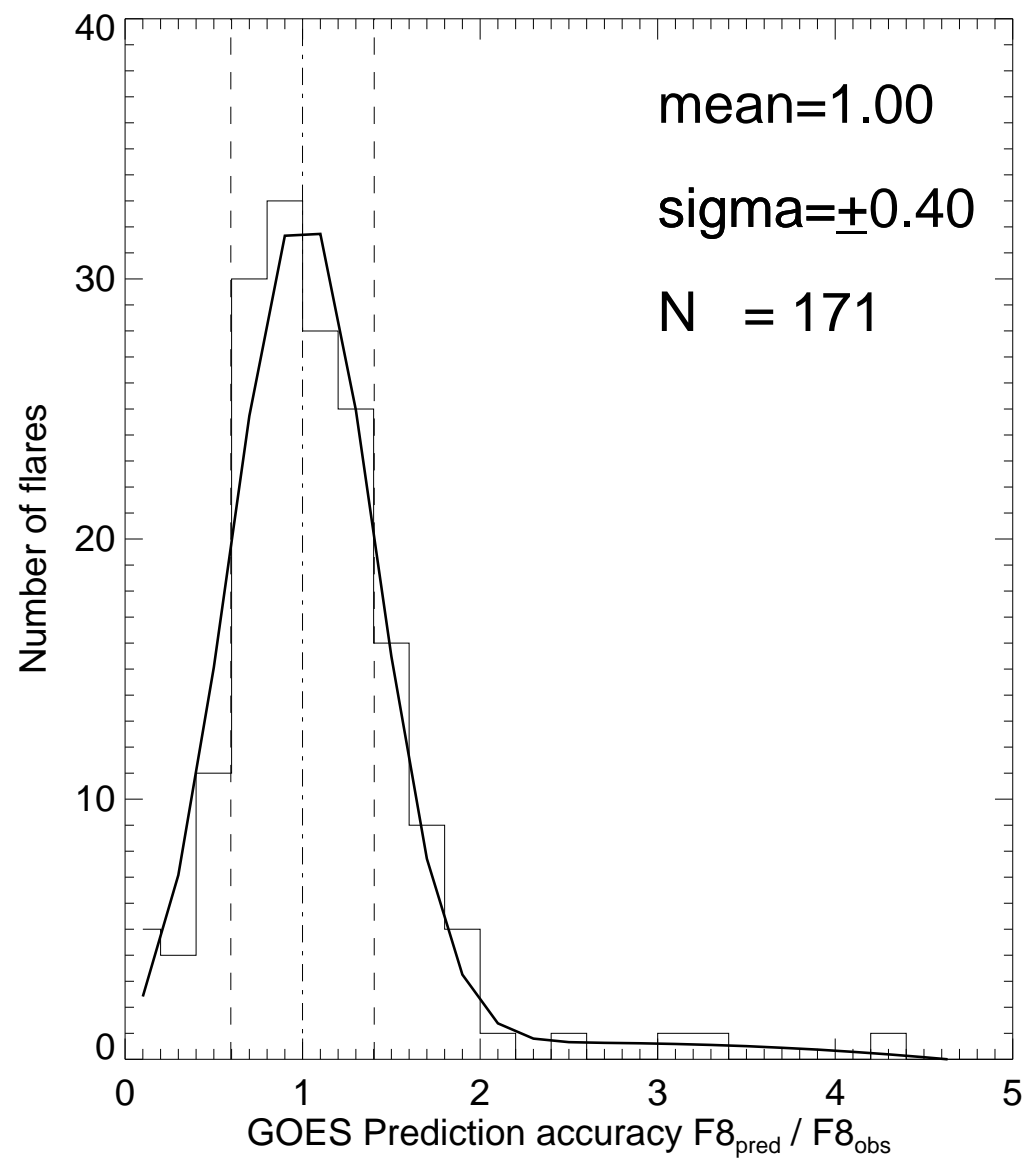

Figure 3. Histogram of the GOES class prediction accuracy, which is specified by the ratio of $q_{a c c}=F 8_{\text {pred }} / F 8_{\text {obs }}$ with a mean and standard deviation of $q_{a c c}=1.0 \pm 0.4$, shown as Gaussian fit. 

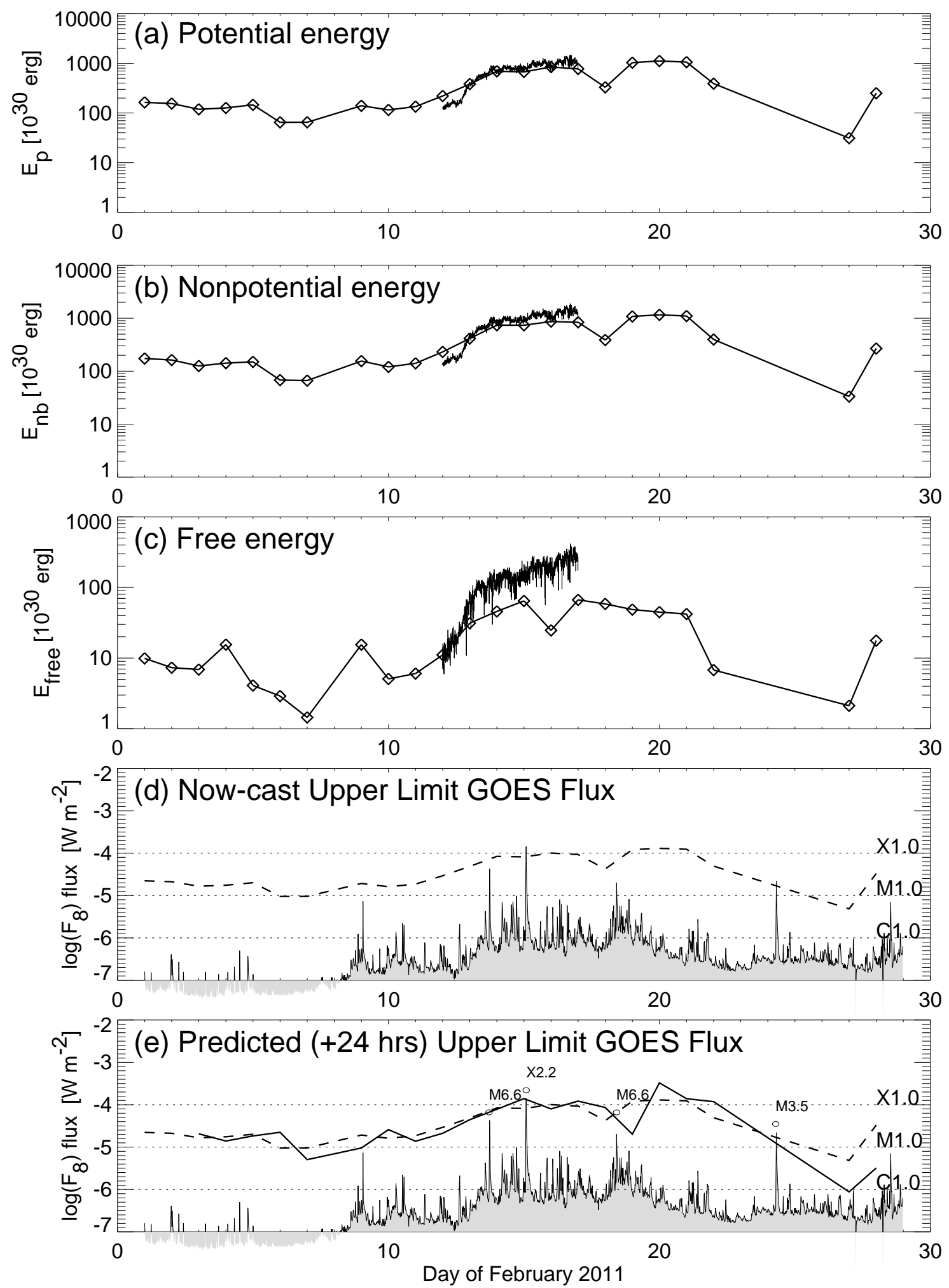

Figure 4. Time evolution of the potential-field energy $E_{p}(\mathrm{a})$, the non-potential energy $E_{n p}(\mathrm{~b})$, the free energy $E_{f r e e}=E_{n p}-E_{p}$ (c) during the month of February 2011, with a daily cadence. The light curves (diamonds) are new calculations with the latest version of the VCA-NLFFF code, while the noisy curves with cadence of 6 minutes are taken from an older version (Aschwanden 2016). Panel (d) shows the GOES light curve (shaded with gray), with a cadence of 0.3 hrs, and the now-cast upper limit of the GOES flux (dashed curve) calculated from the scaling relationship $F_{8}(t) \propto E_{p}(t)^{0.92}$. Panel (e) shows the predicted upper limit of the GOES 1-8 A flux, extrapolated for a time window of +24 hours (solid curve), along with the now-cast predictions (dashed curve). Four observed flare events of GOES class $>M 3.0$ are marked with circles, which all are close to the predicted upper limit of the GOES flux. 